\title{
Basic Guidelines for the Treatment of HIV/HVC Co-Infection
}

\author{
Edgard De Bortholi \\ Emílio Ribas Institute of Infectious Diseases; São Paulo, SP, Brazil
}

\begin{abstract}
The treatment of HIV/HCV co-infection presents many questions that have not yet been answered or on which there is no consensus. Since it is a recently introduced issue, the guidelines in the literature are divergent on some points. The still fragmented knowledge and lack of long-term worldwide experience in the treatment of such co-infections has forced referral facilities to constantly update their approaches.

Some guidelines for the management of the HIV/HCV coinfected patient have been proposed, and new recommendations are particularly necessary:
\end{abstract}

- Management of patients with persistently normal aminotransferase levels.

- Definition and quantification of liver fibrosis: when and how?

- Predictors of the response to anti-HCV therapy in coinfected patients.

- Therapeutic doses of pegylated interferon and ribavirin.

- Treatment duration.

- Treatment of nonresponsive and recidivist patients.

- Treatment of acute infection in HIV-positive patients.

- HIV/HCV/HBV co-infected patients.

- Interaction between antiretroviral drugs and anti-HCV therapeutics.

- Antiretroviral hepatotoxicity in co-infected patients.

- Antiretroviral drugs and recommended doses in hepatic insufficiency.

\section{Management of Patients with Persistently Normal Aminotransferase}

Establishing the persistence of normal aminotransferase levels in HCV-infected patients is difficult, especially in coinfected patients. Fluctuations in the levels of aspartate aminotransferase and alanine aminotransferase are common in this group of patients due to several factors, among which are the use of drugs of hepatotoxic potential, alcohol abuse, and infection with other opportunistic agents. In contrast to mono-infected patients, who present persistently normal alanine aminotransferase levels ( $25 \%)$, co-infected patients present levels of 7-9\%. However, of such patients, $25-40 \%$ present advanced liver fibrosis, which leads to liver cirrhosis.

The rapid evolution of fibrosis in co-infected patients, even in those with normal transaminase levels, indicates treatment, based on patient motivation, duration of the disease, fibrosis stage, and viral load of HCV.

The Brazilian Journal of Infectious Diseases 2007;11 (5) Suppl. 1:38-42. (C) 2007 by The Brazilian Journal of Infectious Diseases and Contexto Publishing. All rights reserved.

\section{Definition and Quantification of Liver Fibrosis: When and How?}

Various studies have demonstrated the rapidity of liver fibrosis progression in HIV/HCV co-infected patients. Such patients, even those presenting little or no fibrosis, should undergo histological evaluation at least every two years.

Unfortunately, since it is an invasive procedure, liver biopsy might present complications resulting from technical performance. Pathologist reports are often made difficult by the small size of the liver fragments obtained in the biopsy, which has repercussions for the indication of anti-HCV therapy.

Despite the disadvantages of liver biopsy, it remains the principal technique for determining the severity of hepatic injury.

Noninvasive procedures to assess the level of the liver fibrosis are currently divided into two categories: imaging techniques, such as elastometry (FibroScan); and the use of biochemical markers (Fibrotest, APRISHASTA, FIB-4, and Forn Index). These procedures are accurate at discriminating between the absence of fibrosis and advanced fibrosis but are not very precise at evaluating the intermediate stages of the fibrosis. They present good predictive value for advanced liver fibrosis and cirrhosis.

Biochemical marker determination in co-infected patients is of little utility, given the inflammatory nature of the HIV disease and the fact that, in this population, hepatotoxic drugs are used. Such drugs interfere with the serum markers of fibrosis in various ways: atazanavir elevates bilirubin levels; non-nucleoside analog reverse transcriptase inhibitors affect gamma glutamyl-transferase levels; and some protease inhibitors elevate cholesterol levels.

Due to technical difficulties, complications and the number of times that the degree of fibrosis must be determined, liver biopsy might soon be replaced by FibroScan for the evaluation of co-infected patients, at least in one of their follow-up visits.

\section{Predictors of the Response to Anti-HCV Therapy in Co- Infected Patients}

The current trend in the treatment of HCV infection is to individualize the approach.

All HIV/HCV co-infected patients should undergo HCV genotyping before any therapeutic decision is made.

Since the first attempts at treatment of HCV infection in HIV-positive patients, a small percentage of patients presenting a sustained virological response at the end of the treatment has been demonstrated, especially those with manifested immunosuppression with low CD4 counts. These patients present high viral loads of HCV, lower CD8 anti-HCV responses, greater frequency of liver steatosis caused by the 
use of alcohol and hepatotoxic drugs (resulting from a lower kinetic response to HCV treatment), a higher percentage of adverse effects, and worse treatment compliance.

In order to achieve an early virological response, it is necessary to interfere with the natural and adaptive responses, which are reduced in co-infected patients, inducing a delay in viral clearance, which translates to a late virological response. High levels of HCV RNA in co-infected patients might also explain the low rate at which a sustained virological response is achieved.

Approximately 30\% of co-infected patients do not present a significant reduction in HCV viremia during the first month of treatment with pegylated interferon and ribavirin.

The precise indication for treatment in HIV/HCV coinfected patients is CD4 counts greater than 350 cells $/ \mathrm{mm}^{3}$. In patients with CD4 counts between 200 and 350 cells $/ \mathrm{mm}^{3}$, the decision to treat the HCV infection should take other factors into account, such as the duration of the HCV infection, the severity of the liver disease, the degree of HIV suppression, and the classic predictors of the response to the HCV treatment (genotype and viral load).

A sustained virological response can be predicted based on the HCV RNA serum negativity in week 4 of treatment. However, a reduction of less than $2 \log \mathrm{IU} / \mathrm{mL}$ in the viral load of HCV in week 12 or the presence of detectable viremia in week 24 predicts the absence of a virological response, and the treatment should be discontinued.

Ongoing studies focus on the maintenance of the treatment for 72 weeks in co-infected patients who are nonresponsive at week 24. Perhaps this, or even a longer time with smaller and fractionated doses, is the real treatment time for HIVpositive patients.

\section{Contraindications and Special Populations}

The patients with hepatic decompensation (ascites, digestive bleeding, hepatic encephalopathy, etc.) cannot be treated with interferon due to the high risk of developing serious complications. In these patients, the possibility of a liver transplant should be considered, even though this possibility is still remote because of the inherent difficulties of immunosuppression and HIV infection.

However, the patients with compensated (Child-Pugh class A or B) cirrhosis can be treated since they are the ones that benefit most from the treatment.

As to intravenous drug users and chronic alcoholics, the treatment should be postponed until the habits are controlled, and such patients should be referred to a detoxification program.

\section{Therapeutic Doses of Pegylated Interferon and Ribavirin}

To date, the efficacy of high doses of pegylated interferon in the treatment of HIV/HCV co-infected patients has not been confirmed, and we should therefore await the results of future investigations.

Ribavirin induces errors in the viral replication cycle, and this effect is relevant, principally in HIV-positive patients, in whom the mediated immune response is impaired. Various authors have demonstrated that the dose of ribavirin is directly related to achieving a sustained virological response. High doses of this drug are fundamental to the maintenance of viral suppression, especially in the first weeks of the treatment. Therefore, the recommended dose for the treatment of $\mathrm{HCV}$ is $15 \mathrm{mg} / \mathrm{kg} /$ day (the minimal dose for adjustment is $11 \mathrm{mg} / \mathrm{kg} /$ dose).

\section{Treatment Duration}

The current consensuses recommend that the duration of treatment for $\mathrm{HIV} / \mathrm{HCV}$ co-infected patients be 48 weeks regardless of the genotype. However, values of HCV RNA > 2 $\log \mathrm{IU} / \mathrm{mL}$ at week 12 have a negative predictive value similar to that observed for mono-infected patients. Nevertheless, recent studies have questioned this simplistic view of the treatment time. When patients infected with genotype 2 or 3 test negative for HCV RNA in week 4 and maintain their negativity until week 12, the treatment time is reduced to 24 weeks. When patients infected with genotype 1 or 4 are in the same situation, the duration of treatment is extended to 48 weeks. For the patients who, despite positivity in week 4, present a drop in HCV RNA > $2 \log$ IU/mL by week 12, the polymerase chain reaction (PCR) for qualitative HCV should be repeated in week 12. If the PCR is negative, and the genotype is 2 or 3 , the treatment should continue to week 48. However, if the genotype is 1 or 4 , the treatment should be maintained until week 72 .

If the qualitative PCR for HCV is still positive at week 24, or there is an HCV RNA reduction $<2 \log \mathrm{IU} / \mathrm{mL}$ in week 12, the treatment should be discontinued, regardless of the genotype.

Using erythropoietin and filgrastim has been a good strategy, even in co-infected patients, for the treatment of anemia and neutropenia, respectively.

\section{Treatment of Nonresponsive and Recidivist Patients}

At least to date, the HIV/HCV co-infected patients, in contrast with mono-infected patients, are not candidates for liver transplant, due to the disease progression and the absence of a therapeutic response.

A growing number of co-infected patients who have already undergone treatment with standard interferon with or without ribavirin, especially those with progression of the fibrosis staging, can now be retreated with pegylated interferon and ribavirin.

For the patients who have already used pegylated interferon and ribavirin and did not respond to the treatment or presented recurrence, there is currently no regimen for infection control.

Despite the absence of a virological response in these patients, $35-43 \%$ of them present a reduction in the degree of liver fibrosis, demonstrating the antifibrotic effect of interferon. These data provide a rationale if the maintenance of interferon, even in small doses, for a prolonged time, has no effect on the progression of the fibrosis, even in the patients in whom HCV has not been eradicated. 
New anti-HCV drugs are urgently needed, especially for this group of patients, who depend exclusively on clinical treatment.

\section{Treatment of Acute Infection in HIV-Positive Patients}

Outbreaks of acute HCV infection in homosexuals have been reported in some European cities. Despite the knowledge of the low transmissibility of HCV through sex, sexual practices that generate traumatic lesions and genital ulcerations have been associated with the infection.

The natural history of HCV infection in HIV patients has demonstrated its evolution to chronicity. Therefore, early therapeutic intervention (in the acute phase of infection) is particularly indicated in these cases, although treatment should not be instituted earlier than 12 weeks after the exposure, due to the possibility of spontaneous viral clearance. However, a delay in starting treatment might result in a reduction of the therapeutic response.

The treatment of acute HCV infection in HIV-positive patients seems to provide a pattern of lower virological response when compared to HIV-negative patients. However, the viral clearance pattern obtained in HIV-positive patients in the acute phase is greater than that seen in those presenting chronic infection.

Acute infection in HIV-positive patients should be treated with pegylated interferon and ribavirin for 24 weeks.

\section{HIV/HCV/HBV Co-Infected Patients}

In HIV-positive patients living in developed countries, the prevalence of multiple viral hepatitis(HBV/VCV; VBV/VDV; VBV/ $\mathrm{HCV} / \mathrm{HDV}$ ) is lower than $3 \%$. However, this is still higher than that seen in the general population. Patients presenting HBV/ HCV co-infection seem to present reciprocal inhibition of viral replication, with one of the viruses being predominant. However, this predominance might oscillate from one virus to the other. Nevertheless, in patients with severe immunosuppression, replication of all of the viruses might occur simultaneously. In HIV-positive patients with good immune status, the interference seems to favor HCV, to the detriment of HBV.

The progression of the liver disease seems to be more accelerated in HIV-positive patients infected with both HBV and HCV. In addition, these individuals are more likely to develop hepatocarcinoma.

Using interferon to treat chronic HDV in HIV-positive patients is rarely effective.

There is no established consensus as to the approach to treating multiple viral hepatitis. Few studies have examined the efficacy and safety of the combination of pegylated interferon and ribavirin for treating multiple infections in HIVpositive patients. When possible, all of the viruses involved in the hepatitis should be treated.

\section{Interaction between Antiretroviral Medications and Anti- HCV Therapy}

The highly active antiretroviral therapy (HAART) regimen is associated with increased survival due to the fact that it controls HIV and slows the progression of the hepatic disease. The better prognosis that the HAART regimen confers on HIV/ HCV co-infected patients is probably due to deceleration of the progression of hepatic fibrosis. This has principally been demonstrated in HIV patients treated with protease inhibitors.

Some studies have suggested the early introduction of antiretroviral therapy in HIV/HCV co-infected patients, considering the beginning of the HAART regimen in patients with CD4 counts $>350$ cells $/ \mathrm{mm}^{3}$, particularly in male patients and in those who acquired HCV at an advanced age, which would decrease the effects resulting from immune reconstitution and the slowing of the progression of HCVinduced hepatic disease.

The principal complication in the treatment of HIV-infected patients is the interaction between ribavirin and some antiretroviral drugs.

Ribavirin can decrease the concentrations of some nucleoside analog reverse transcriptase inhibitors (NRTIs), although there seem to be no clinical consequences.

The adverse effects of the NRTIs have been associated with HCV co-infection, female gender, obesity, and prolonged exposure to these drugs. The principal NRTI combinations associated with lower impact on the liver are as follows: lamivudine+abacavir (3TC/ABC); 3TC+zidovudine (AZT); 3TC+ABC+AZT; and emtricitabine + tenofovir. However, recent studies have indicated that ribavirin interferes with the effects of $A B C$, and, therefore, its use in the HAART regimen for HCV patients should be considered with caution.

The greatest toxicity is that resulting from the concomitant use of didanosine and ribavirin, worsening with the addition of stavudine. These combinations increase the possibility of mitochondrial toxicity and are potentiated by the presence of HIV and HCV. In patients treated with these antiretroviral drugs combined with ribavirin, lactic acidosis, pancreatitis and hepatic decompensation have been reported. Therefore, it is recommended that replacements for these antiretroviral drugs be found for patients who will initiate the treatment for HCV. In addition, AZT should be replaced, when possible, due to frequent, severe anemia and neutropenia when AZT is administered together with ribavirin.

It is important to emphasize that the antiretroviral drugs, as well as the drugs used in the treatment and prophylaxis of the various opportunistic infections, are metabolized by the liver and, therefore, many of these metabolites can increase the risk of hepatic lesion.

The chart below shows the principal interactions between the drugs used, facilitating the choice of antiretroviral drugs in the clinical approach to co-infected patients.

The combination of ritonavir and saquinavir has proven to be highly hepatotoxic, and its use is not currently recommended, especially in HIV/HCV co-infected patients with concomitant tuberculosis, in whom noncompliance with treatment and, principally, the elevation of transaminase levels result from the discontinuation of the treatment with antituberculosis drugs or the introduction of alternative 
Chart 1. Drug interactions

\begin{tabular}{|c|c|c|c|}
\hline Antiretroviral drugs & Adverse effects & Therapeutic alternative & Interaction with anti-HCV therapy \\
\hline Zidovudine & Anemia and neutropenia & Stavudine & $\begin{array}{l}\text { Potentiates the probability of anemia } \\
\text { caused by ribavirin }\end{array}$ \\
\hline Didanosine & $\begin{array}{l}\text { Hepatotoxicity } \\
\text { Mitochondrial toxicity }\end{array}$ & Lamivudine or efavirenz & $\begin{array}{l}\text { Ribavirin inhibits the } \\
\text { phosphorylation of this drug }\end{array}$ \\
\hline Stavudine & $\begin{array}{l}\text { Hepatotoxicity } \\
\text { Mitochondrial toxicity }\end{array}$ & Lamivudine or efavirenz & $\begin{array}{l}\text { Should not be combined with } \\
\text { didanosine due to potentiation of } \\
\text { mitochondrial toxicity }\end{array}$ \\
\hline Nevirapine & Hepatotoxicity & Efavirenz & $\begin{array}{l}\text { Do not combine with didanosine and } \\
\text { stavudine; potentiation of } \\
\text { mitochondrial toxicity }\end{array}$ \\
\hline Ribavirin & Hepatotoxicity & $\begin{array}{l}\text { Tolerated in low doses } \\
\text { when used in combination } \\
\text { with other protease inhibitors }\end{array}$ & $\begin{array}{l}\text { Increases the probability of } \\
\text { intolerance to the antiretroviral } \\
\text { therapy. }\end{array}$ \\
\hline
\end{tabular}

HCV=hepatitis C virus.

Chart 2. Antiretrovirals and recommended doses in liver failure

\begin{tabular}{|c|c|c|}
\hline Name & Hepatic metabolism & Recommendations \\
\hline \multicolumn{3}{|l|}{ NRTIs } \\
\hline Abacavir & Yes & Mild $\left(A^{*}\right)=200$ mg twice a day / moderate/severe=contraindicated \\
\hline Didanosine & Yes & Not recommended \\
\hline Emtricitabine & No & No need for dose adjustment -recommended \\
\hline Lamivudine & No & No need for dose adjustment -recommended \\
\hline Stavudine & Unknown & No need for dose adjustment -recommended \\
\hline Zalcitabine & No & No need for dose adjustment -recommended \\
\hline Zidovudine & Yes & No need for dose adjustment -recommended \\
\hline \multicolumn{3}{|r|}{ 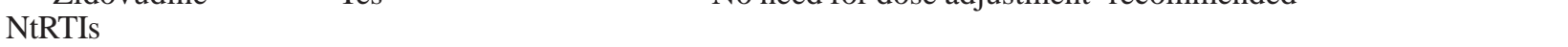 } \\
\hline Tenofovir & No & No need for dose adjustment -recommended \\
\hline \multicolumn{3}{|r|}{ 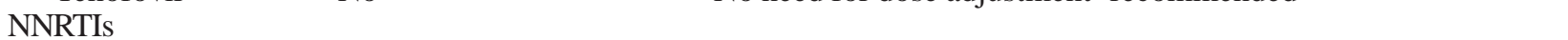 } \\
\hline Delavirdine & Yes & Not recommended \\
\hline Efavirenz & Yes & Not recommended \\
\hline Nevirapine & Yes & $\begin{array}{l}\text { Mild }\left(\mathrm{A}^{*}\right) / \text { moderate }\left(\mathrm{B}^{*}\right)=\text { No need for dose adjustment-recommended } \\
\text { Severe }\left(\mathrm{C}^{*}\right)=\text { contraindicated }\end{array}$ \\
\hline \multicolumn{3}{|r|}{ 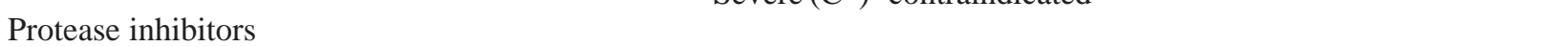 } \\
\hline Atazanavir & Yes & $\begin{array}{l}\text { Mild }\left(A^{*}\right)=\text { No need for dose adjustment }- \text { recommended } \\
\text { Moderate }\left(B^{*}\right)=300 \mathrm{mg} / \text { day. Severe }\left(C^{*}\right)=\text { contraindicated }\end{array}$ \\
\hline Fosamprenavir & Yes & $\begin{array}{l}\text { Mild/moderate }=700 \mathrm{mg} 2 \text { xday } \\
\text { Severe }=\text { contraindicated (because the dose cannot be reduced to } \\
\text { lower than } 700 \mathrm{mg} \text { ) }\end{array}$ \\
\hline Indinavir & Yes & Mild/moderate=600 mg 3× day. Severe=not recommended \\
\hline Nelfinavir & Yes & Not recommended \\
\hline Ritonavir & Yes & $\begin{array}{l}\text { Mild/moderate=No need for dose adjustment } \text {-recommended } \\
\text { Severe=not recommended }\end{array}$ \\
\hline Saquinavir & Yes & Mild/ moderate=not recommended. Severe $=$ contraindicated \\
\hline \multicolumn{3}{|c|}{ Boosters w/ ritonavir } \\
\hline Atazanavir/ritonavir & Yes & $\begin{array}{l}\text { Not recommended at some levels of hepatic insufficiency because } \\
\text { it has not been adequately studied }\end{array}$ \\
\hline \multicolumn{2}{|c|}{ Fosamprenavir/ritonavir } & Yes Not recommended \\
\hline Lopinavir/ritonavir & Yes & Not recommended \\
\hline Saquinavir/ritonavir & Yes & Mild $/$ moderate $=$ Not recommended. Severe $=$ contraindicated \\
\hline Tipranavir/ritonavir & Yes & $\begin{array}{l}\text { Mild=No need for dose adjustment - recommended } \\
\text { Moderate/severe }=\text { contraindicated }\end{array}$ \\
\hline \multicolumn{3}{|l|}{ Fusion inhibitors } \\
\hline Enfuvirtide & Unknown & nended \\
\hline
\end{tabular}

*Child-Pugh class. NRTIs=nucleoside analog reverse transcriptase inhibitors; NtRTIs=nucleotide analog reverse transcriptase inhibitors; NNRTIs=non-nucleoside analog reverse transcriptase inhibitors. 
regimens that are less efficient in the resolution of infection with Mycobacterium tuberculosis.

The data currently available are insufficient to determine the nature of the interaction of atazanavir, tenofovir, fosamprenavir, and tipranavir with the therapeutic regimen in HCV infection.

\section{References}

1. Arends J.E., Boucher C.A.B., Hoepelman A.I.M. Hepatitis C virus and human imunodeficiency virus coinfected: where do we stand? Journal of Medicine 2005;63:156-63.
2. Lai, et al. Antiretroviral medication considerations for individuals coinfected with HIV and hepatitis C virus. AIDS Patient Care and STDs 2006;20(10):678-92.

3. Rockstroh J.K. Influence of viral hepatitis on HIV infection. Journal of Hepatology 2006;44:S25-S7.

4. Sancho A.R., Soriano Vicente. Coinfección por el VIH y el virus de la hepatitis C. http//www.doyma. Acessado em 26/02/2007.

5. Soriano V., et al. Care of patients coinfected with HIV and hepatitis C virus: 2007 updated recomendations from the HCV-HIV international panel. AIDS 2007,21:1073-89.

6. Thomas L.D. Options for treatment of hepatitis C in HIV-infected persons. Journal of Hepatology 2006;44:S40-S3. 